\title{
Interference Study of Micro Licensing for 5G Micro Operator Small Cell Deployments
}

\author{
Marja Matinmikko ${ }^{1}$, Antti Roivainen ${ }^{2}$, Matti Latva-aho ${ }^{1}$, Kimmo Hiltunen $^{1}$ \\ ${ }^{1}$ Centre for Wireless Communications (CWC), University of Oulu, Finland \\ \{marja.matinmikko, matti.latva-aho, kimmo.hiltunen\}@oulu.fi \\ ${ }^{2}$ Keysight Technologies Finland Oy, Finland \\ antti.roivainen@keysight.com
}

\begin{abstract}
G brings along very dense small cell deployments in specific locations such as hospitals, campuses, shopping malls, and factories. This will result in a novel 5G deployment scenario where different stakeholders, i.e., micro operators, are issued local spectrum access rights in the form of micro licenses, to deploy networks in the specific premises. This new form of sharing-based micro licensing guarantees that the local $5 \mathrm{G}$ networks remain free from harmful interference from each other and also protects potential incumbent spectrum users' rights. It admits a larger number of stakeholders to gain access to the $5 \mathrm{G}$ spectrum to serve different vertical sectors' needs beyond traditional mobile network operators (MNO) improving the competition landscape. We characterize the resulting interference scenarios between the different micro operators' deployments and focus on the building-to-building scenario where two micro operators hold micro licenses in separate buildings in co-channel and adjacent channel cases. We analyze the resulting allowable transmit power levels of a base station from inside one building towards an end user mobile terminal inside another building as a function of the minimum separation distance between the two micro operator networks. Numerical results are provided for the example case of the $3.5 \mathrm{GHz}$ band with different building entry losses characterizing the impact of propagation characteristics on the resulting interference levels. The results indicate that the building entry losses strongly influence the interference levels and resulting required minimum separation distances, which calls for flexibility in determining the micro license conditions for the building specific situation.
\end{abstract}

Keywords: 5G, interference management, micro operator, spectrum sharing

\section{Introduction}

Next generation mobile communication networks known as $5 \mathrm{G}$ are expected to drive industrial and societal transformations and economic growth with high quality mobile broadband offerings and supporting of new types of high demand applications. Provisioning of high quality wireless connectivity in specific locations such as schools, transport hubs, public service providers' units, and enterprises has become a key societal objective in Europe as the enabler for new services [1]. The location specific needs 
for wireless connectivity have become a major design criteria in the development of 5G networks and their applications in different vertical sectors [2]. There is a growing need for locally operated wireless network deployments by different stakeholders to meet ever increasing requirements for higher capacity, higher data rate, lower latency, massive device density, and reduced capital and operational costs as discussed in [3].

Timely availability of $5 \mathrm{G}$ spectrum will be critical for the roll out of the new networks. These networks are expected to be deployed in a wide range of frequency bands with different propagation characteristics including existing spectrum bands for mobile below $1 \mathrm{GHz}$ and between $1-6 \mathrm{GHz}$, as well as new spectrum above $6 \mathrm{GHz}$ especially in the millimeter wave range $(24-86 \mathrm{GHz})$ as expected from the World Radiocomunication Conference in 2019 (WRC-19). While regulators are globally committed to making new $5 \mathrm{G}$ spectrum available, the authorization models to use the $5 \mathrm{G}$ spectrum is an open topic and will include a mix of licensed and unlicensed models [1], [4]. New sharing based spectrum authorization models for granting access rights to use the $5 \mathrm{G}$ bands among the applicants in an objective, transparent, non-discriminatory and proportionate way are urgently needed to support innovation and market entry as outlined in [4].

Several spectrum sharing models have been introduced in regulation to consider different spectrum authorization regimes to admit additional users while protecting the incumbents in the band. European Licensed Shared Access (LSA) discussed in the 2.32.4 GHz and 3.6-3.8 GHz bands introduces additional licensed users while protecting the existing incumbent users and giving quality of service guarantees also for the new LSA license holders [5]. However, the authorization model for granting the LSA licenses is determined to be a national matter left for the national regulators and has not been discussed yet. A three-tier sharing model for Citizens Broadband Radio Service (CBRS) by the Federal Communications Commission (FCC) in US introduces two layers of additional users under licensed or general authorization regimes in the 3.55-3.7 $\mathrm{GHz}$ band while protecting the incumbents [6]. The priority access layers (PAL) introduces new local spectrum licenses of $10 \mathrm{MHz}$ bandwidth to be granted via auctions for three-year license period over a geographic area of census tract. Both the LSA and CBRS models introduce additional local users since country-wide spectrum availability cannot be guaranteed due to incumbent activity that needs to be protected.

In the research domain, there have been studies on sharing between mobile network operators (MNO) in a so called co-primary shared model [7] where several MNOs access a common band with equal access rights. This model has also been considered for the millimeter wave bands in [8]-[9] requiring either complex coordination mechanisms between MNOs or resulting in interference between them. In particular, new sharingbased local spectrum authorization models are needed for ultra-dense small cell deployments in specific buildings where venue owners role becomes increasingly important as discussed in [10]. To this end, the development of suitable spectrum authorization models for $5 \mathrm{G}$ is an open topic in terms of the level of exclusivity in spectrum use and resulting mechanisms for protection from harmful interference. The use of higher carrier frequencies for $5 \mathrm{G}$ inherently assumes local network deployments due the propagation characteristics, which needs to be properly taken into account in designing the authorization mechanisms. 
There is a growing pressure to preserve a competitive environment open the mobile connectivity market to new entrants [1]. Future $5 \mathrm{G}$ networks will be able to share various resources establishing end-to-end network slices particularly in the higher frequency bands where the new networks will consist of very dense deployments of small cells connected with high capacity backhauls [2]. The concept of micro operators as new entrants to the mobile market were discussed in [3] and [12] to establish local network deployments to complement traditional MNO offerings. Their appearance is highly dependent on the local availability of $5 \mathrm{G}$ spectrum and the ability to lease the required parts of the infrastructure on-demand as a service without high upfront investments.

In the development of new spectrum sharing and authorization models for $5 \mathrm{G}$, interference characterization between the systems involved in sharing is critical and requires proper modeling of the wireless propagation characteristics in the specific frequency bands and deployment scenarios. This paper introduces a novel deployment scenario for $5 \mathrm{G}$ where local indoor small cell networks are deployed in separate buildings by different micro operators with locally issued spectrum micro licenses. Interference scenarios between the two different operators having local indoor small cell deployments in co-channel and adjacent channel cases are modeled and numerical examples of allowed interfering power levels are calculated in the $3.5 \mathrm{GHz}$ band.

The rest of this paper is organized as follows. In Section II we introduce the system model of micro operator indoor small cell networks with micro licensing and the resulting interference scenarios. Section III presents the interference calculation methodology, followed by numerical results in Section IV. Finally, conclusions are drawn in Section V together with future research directions.

\section{$2 \quad$ Local micro operator deployments}

$5 \mathrm{G}$ will introduce small cell deployments that will serve the versatile needs of different vertical sectors as described in [2]. These local cellular network deployments could in the future be operated by different stakeholders in addition to the currently dominant MNOs. In this section we present the new micro operator concept described in [3] for the establishment of local small cell deployments and characterize the resulting interference scenarios between the different micro operator networks as outlined in Fig. 1.

\subsection{Micro operator concept}

The authorization mechanisms to assign spectrum access rights to those requesting it are in the key position to shape the future $5 \mathrm{G}$ mobile communication market. Traditional spectrum authorization models for providing mobile services include granting of individual access rights typically through auctions leading to a small number of MNOs to deploy nation-wide networks with high infrastructure investments. Currently, the only option for non-MNOs to deploy local networks is through the general authorization (unlicensed) model for the establishment of wireless local area networks (WLAN) 
without quality guarantees. These models will need to be rethought in $5 \mathrm{G}$ as these networks are envisaged to operate also in considerably higher carrier frequencies with smaller coverage areas, which calls for new sharing based spectrum authorization models.

The concept of micro operators was recently proposed in [3] and [12] to allow different stakeholders to establish locally operated small cell networks in various places such as shopping malls, hospitals, sports arenas, and industry plants based on local spectrum availability. Since these different deployment areas require high-quality guaranteed wireless connectivity, which is only possible when operations are free from harmful interference from other wireless systems, it is justified that the micro operators obtain spectrum micro licenses with local exclusive access rights to deploy and operate small cell 5G networks in a specific location for a given license duration. This model presents a major paradigm shift in spectrum authorization by combining the benefits of both exclusive licensing and unlicensed models by allowing a larger number of stakeholders to get quality guaranteed local spectrum access rights. The proposed micro licenses can become a key enabler to allow various stakeholders taking up the micro operator role to deploy $5 \mathrm{G}$ small cell networks in specific high-demand areas to serve different vertical sectors' needs. These new micro operators can provide a wide variety of tailored services in specific locations to complement traditional mobile broadband offerings to realize the $5 \mathrm{G}$ deployment plans set, e.g., in [4].

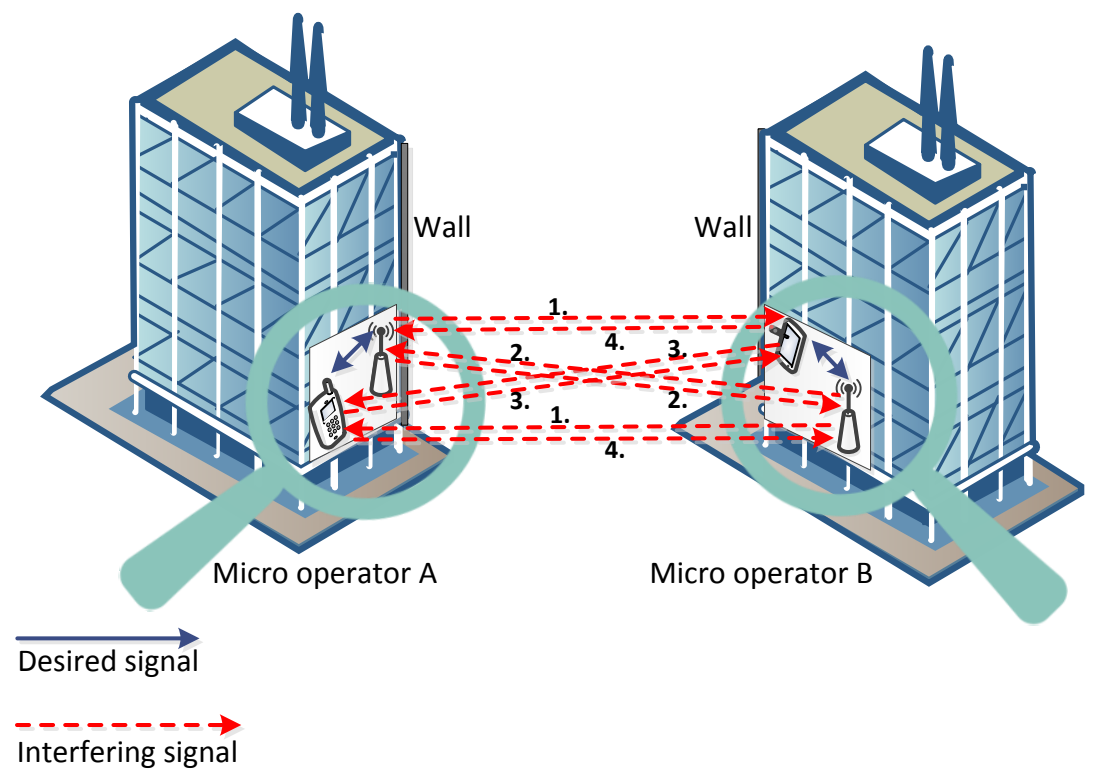

Fig. 1. Micro operator deployments in adjacent buildings and resulting interference scenarios. 


\subsection{Interference characterization}

Industry, regulators and MNOs are seeking for spectrum for $5 \mathrm{G}$ networks from various frequency ranges including existing bands below $1 \mathrm{GHz}$ and between $1-6 \mathrm{GHz}$ as well as new bands above $6 \mathrm{GHz}$ [4]. For the regulators to allow 5G networks to access the bands, they will need to carefully conduct sharing and coexistence studies to ensure the feasibility of sharing between entrant 5G networks and incumbent systems as well as between the entrant system deployments in co-channel and adjacent channel cases. In these studies, interference characterization between the different systems is critical, which in turn requires proper propagation modeling and characterization of the systems involved in spectrum sharing.

In cellular deployments, the macro and micro base stations have typically larger transmission power and antenna gain than indoor base stations resulting in higher coverage areas to serve the mobile terminals. In the worst case where the different MNOs are exploiting the same or adjacent frequency bands, this leads to extremely harmful interference and long separation distances between networks in outdoor environment. In order for two operators to deploy their networks close to each other, the interference caused by one network on the other must be at pre-define tolerable level.

The proposed locally issued spectrum micro licenses require interference coordination between the different license holders and potential incumbent spectrum users in the band to guarantee that their operations remain free from harmful interference. Spectrum sharing (i.e., co-channel case) and coexistence (i.e., adjacent channel case) between different micro license holders requires identification of the different interference scenarios to develop rules and conditions for the micro licensing that guarantee that the networks remain free from harmful interference. Fig. 1 illustrates a simplified interference scenario where two micro operators A and B are granted micro licenses in different buildings in the same channel to operate their own time division duplex (TDD) small cell indoor networks that are unsynchronous. This results in potential interference from one indoor network in one building to another indoor network in another building and vice versa. These interference scenarios include the following:

1. From base station to mobile terminal (downlink to downlink interference)

2. From base station to base station (downlink to uplink interference)

3. From mobile terminal to mobile terminal (uplink to downlink interference)

4. From mobile terminal to base station (uplink to uplink interference).

In characterizing the resulting interferences between the local $5 \mathrm{G}$ networks deployed by the different micro operators, the modeling of the propagation environment is of great importance. Several radio channel measurement campaigns have been carried out to characterize propagation environment in different frequency bands. For the interference characterization, the most important propagation characteristics are path loss (PL) and shadow fading. Several PL models, e.g., ITU-R models [13], have been reported for various scenarios for the frequency bands below $6 \mathrm{GHz}$. Recently, the extensions of PL models have been proposed for millimeter bands, e.g., in [14]. However, the PL model for building-to-building scenario has not received much attention in the existing research literature. 


\section{Calculation of maximum allowable transmit power}

For two radio systems to operate in the same frequency band geographically close to each other, there is a need to define an interference threshold so that a victim receiver is protected from harmful interference from an interfering transmitter. In case there are two micro licensee deployments run by different micro operators, the protection can be achieved by defining a sufficient separation distance between the interfering transmitter and the victim receiver such that the PL between two systems is high enough.

In this paper we study the maximum allowable transmit power caused by a micro operator that results in protection from harmful interference at another micro operator. We focus on the interference scenario where the transmission from a base station of one micro operator is interfering a victim mobile terminal of another micro operator, and investigate the required minimum separation distance between the two micro operator networks. This interference scenario is selected as it presents a worst case scenario since the transmission power of an base station is higher than an mobile terminal and a victim mobile terminal is more vulnerable to interference than a victim base station. This study serves as the starting point for more complicated interference characterizations in 5G for spectrum sharing between small cell deployments of different operators. The minimum separation distance based calculation methodology has been adopted and derived from [15] and [16]. The minimum required PL including the effect of shadow fading is determined based on minimum coupling loss (MCL) using

$$
M C L_{95}=M C L_{50}+\sigma \cdot \sqrt{ } 2 \cdot \operatorname{erf}^{-1}(2 \cdot 0.95-1),
$$

where erf -1 is the inverse error function, $\sigma$ is the standard deviation of shadow fading, and $M C L_{50}$ is the minimum required median MCL. The isotropic antennas are used for base station and mobile terminal [13]. Therefore, the transmit and receive antenna gains and directivity losses can be ignored in the analysis. Furthermore, the feeder loss of base station is set $0 \mathrm{~dB}$ [17]. Thus, $M C L_{50}$ [15], [16] can be presented as

$$
M C L_{50}=P_{\mathrm{t}}-A C I R-I C-G_{\mathrm{b}},
$$

where $P_{\mathrm{t}}$ is effective transmitted interfering power, $A C I R$ is adjacent channel interference ratio in the case where the operators are deployed in adjacent frequency bands (if the operators are assumed to be serving in the same frequency band, the $A C I R$ can be ignored), $I C$ is interference criterion, i.e., maximum allowable received interference power, and $G_{\mathrm{b}}$ is bandwidth mitigation factor expressed as

$$
G_{\mathrm{b}}=\max \left(0 ; 10 \cdot \log _{10}\left(B_{\mathrm{t}} / B_{\mathrm{r}}\right)\right),
$$

where $B_{\mathrm{t}}$ and $B_{\mathrm{r}}$ are the bandwidths of interfering base station and victim mobile terminal, respectively. Typically, $I C$ is set $6 \mathrm{~dB}$ below receive noise power $N$

$$
I C=N-6 \mathrm{~dB},
$$

where

$$
N=-174+10 \cdot \log _{10}\left(B_{\mathrm{r}}\right)+F
$$


where $F$ is receiver noise figure. Due to lack of PL model in the building-to-building scenario, we carry out the analysis by assuming free space loss for outdoor propagation environment, and that the interfering base station and victim mobile terminal are located close to the external wall. Therefore, the path loss is presented as

$$
P L=-27.55+20 \cdot \log _{10}(f)+20 \cdot \log _{10}(d)+L_{\mathrm{W} 1}+L_{\mathrm{W} 2},
$$

where $f$ is carrier frequency in $\mathrm{MHz}, d$ is the minimum separation distance between interfering base station and victim mobile terminal in meters, and $L_{\mathrm{W} 1}$ and $L_{\mathrm{W} 2}$ are the propagation losses caused by the first and the second wall, respectively. By substituting (6) to the left side of (1), the effective transmitted interfering power, i.e., the allowable transmitted interfering power can be expressed as

$$
P_{\mathrm{t}}=A C I R+P L+I C+G_{\mathrm{B}}-\sigma \cdot \sqrt{ } 2 \cdot \operatorname{erf}^{-1}(2 \cdot 0.95-1) .
$$

\section{$4 \quad$ Results}

Next, we analyze the maximum allowable transmit power levels that result in interference protection at the victim receiver for the example case of $3.5 \mathrm{GHz}$ band. The considered scenario includes interference modeling from a base station of one micro licensee network inside one building towards a mobile terminal of another small cell network inside another building. We use different building penetration losses characterizing the impact of propagation characteristics on the interference levels. The base station and mobile terminal parameters are taken from [17] and presented in Table 1. Due to the lack of propagation channel characterization in the case where the transmitter is located in a different building than the receiver, we use $\sigma$ given in [13] for indoor hotspot scenario. It is worth noting that this parameter affects significantly to the presented results. However, the presented PL models for line-of-sight case have shown small $\sigma$ in the existing literature. Moreover, since we use the free space loss for outdoor propagation and base station and mobile terminal are located close to the walls, the $\sigma$ can be assumed to be realistic.

Four different combinations are considered for wall penetration losses $\left(L_{\mathrm{W} 1}+L_{\mathrm{W} 2}\right)$ : $10 \mathrm{~dB}, 25 \mathrm{~dB}, 40 \mathrm{~dB}$, and $48.1 \mathrm{~dB}$. These attenuation levels can be assumed to be caused by different types of wall construction materials in buildings [13], [18]:

- standard multi-pane glass windows in both buildings ( $2 \times \mathrm{SG}), 5 \mathrm{~dB}$ loss per window,

O standard multi-pane glass window in one building and $20 \mathrm{~dB}$ loss caused by concrete wall in another building, $(\mathrm{SG}+\mathrm{C})$,

o concrete walls in both buildings $(2 \times \mathrm{C})$,

○ infrared reflective glass wall (2 x IG) in both buildings, $24.05 \mathrm{~dB}$ loss per wall.

In this study, the micro operators are assumed to be deployed either in the same frequency band (co-channel) or in the adjacent frequency bands (adjacent channel). In the adjacent channel case, the same bandwidths are assumed for interfering base station 
and victim mobile terminal. Therefore, $G_{\mathrm{B}}$ can be omitted from (7) in the adjacent channel case. In the co-channel case, the bandwidths of interfering base station and victim mobile terminal might be different. This leads to different bandwidth mitigation factors $G_{\mathrm{B}}$. However, the maximum allowable received interference power changes equivalently. Therefore, the number of results in the co-channel case are reduced to the maximum bandwidth of interfering base station and victim mobile terminal.

Parameter ACIR takes into account both the adjacent channel leakage power ratio (ACLR) of the transmitter, assumed to be equal to $45 \mathrm{~dB}$ [19], and the adjacent channel selectivity (ACS) of the receiver, equal to $33 \mathrm{~dB}$ (for bandwidths equal to $5 \mathrm{MHz}$ and $10 \mathrm{MHz})$ or $27 \mathrm{~dB}(20 \mathrm{MHz})$ [20]. With the assumed values, the ACIR becomes equal to $32.7 \mathrm{~dB}(5 \mathrm{MHz}, 10 \mathrm{MHz})$ or $26.9 \mathrm{~dB}(20 \mathrm{MHz})$.

Fig. 2 presents the maximum allowable transmit power as a function of minimum separation distance in the co-channel case. If the construction material of both walls is concrete $(2 \times \mathrm{C})$ and standard transmission power of $24 \mathrm{dBm}$ [17] is assumed for base station, the minimum separation distance between victim mobile terminal and interfering base station must be already over 200 meters. If the wall attenuation is lower, e.g., the wall consists of standard multi-pane glass window, the allowable transmit power is significantly smaller. In other words, the transmit power of the base station resulting in interference at the victim mobile terminal must be reduced or the minimum separation distance between the interfering base station and the victim mobile terminal must be significantly increased. This means that, in practice, two indoor operators in different buildings will not be able to operate in the vicinity with respect to each other if they share the same frequency band.

Table 1. Link parameters used in interference analysis

\begin{tabular}{|l|l|}
\hline Parameter & Value \\
\hline Carrier frequency, $f$ & $3500 \mathrm{MHz}$ \\
\hline Bandwidth, BW & $\begin{array}{l}5 \mathrm{MHz}, 10 \mathrm{MHz}, \text { or } \\
20 \mathrm{MHz}\end{array}$ \\
\hline Feeder loss & $0 \mathrm{~dB}$ \\
\hline $\begin{array}{l}\text { Base station and mobile terminal } \\
\text { antenna type }\end{array}$ & isotropic \\
\hline Adjacent channel interference ratio & $\begin{array}{l}32.7 \mathrm{~dB} \text { for } 5 \mathrm{MHz} \\
\text { (ACIR) }\end{array}$ \\
\hline Mobile terminal noise figure, $F$ & $26.9 \mathrm{~dB}$ for $20 \mathrm{MHz}$ \\
\hline & $9 \mathrm{~dB}$ \\
\hline Mobile terminal noise power, $N$ & $-98 \mathrm{dBm}$ for $5 \mathrm{MHz}$, \\
& $-95 \mathrm{dBm}$ for $10 \mathrm{MHz}$, \\
& $-92 \mathrm{dBm}$ for $20 \mathrm{MHz}$ \\
\hline Standard deviation of shadow fading, $\sigma$ & $4 \mathrm{~dB}$ \\
\hline
\end{tabular}


For the adjacent channel case, the maximum allowable transmit power that results in interference protection at the victim receiver as a function of minimum separation distance is shown in Fig. 3. With a single interfering base station having the transmission power of $24 \mathrm{dBm}$, the minimum separation distances are less than 100 meters already with low wall attenuation levels, i.e., with standard multi-pane glass windows in both buildings ( $2 \mathrm{x}$ SG). If the wall construction materials are standard multi-pane glass window in one building and concrete wall in another building, the allowable transmit power would be larger in the order of $30 \mathrm{dBm}$ when the minimum separation distance is 25 meters or longer.

While the present analysis is conducted only for a single interfering base station, the results in Fig. 3 indicate that there could be several base stations of one micro operator, each having $24 \mathrm{dBm}$ maximum transmission power, without causing harmful interference to the mobile terminal of another micro operator if they are located at least 25 meters away from the mobile terminal. Especially, several base stations could operate simultaneously without interfering with the victim mobile terminal in the case of larger attenuations caused by, e.g., concrete walls in both buildings. This leads to the conclusions that a few base stations of one operator do not cause critical interference to the mobile terminal of another operator if the attenuation caused by walls is $25 \mathrm{~dB}$ or larger and operators are deployed in adjacent frequency bands.

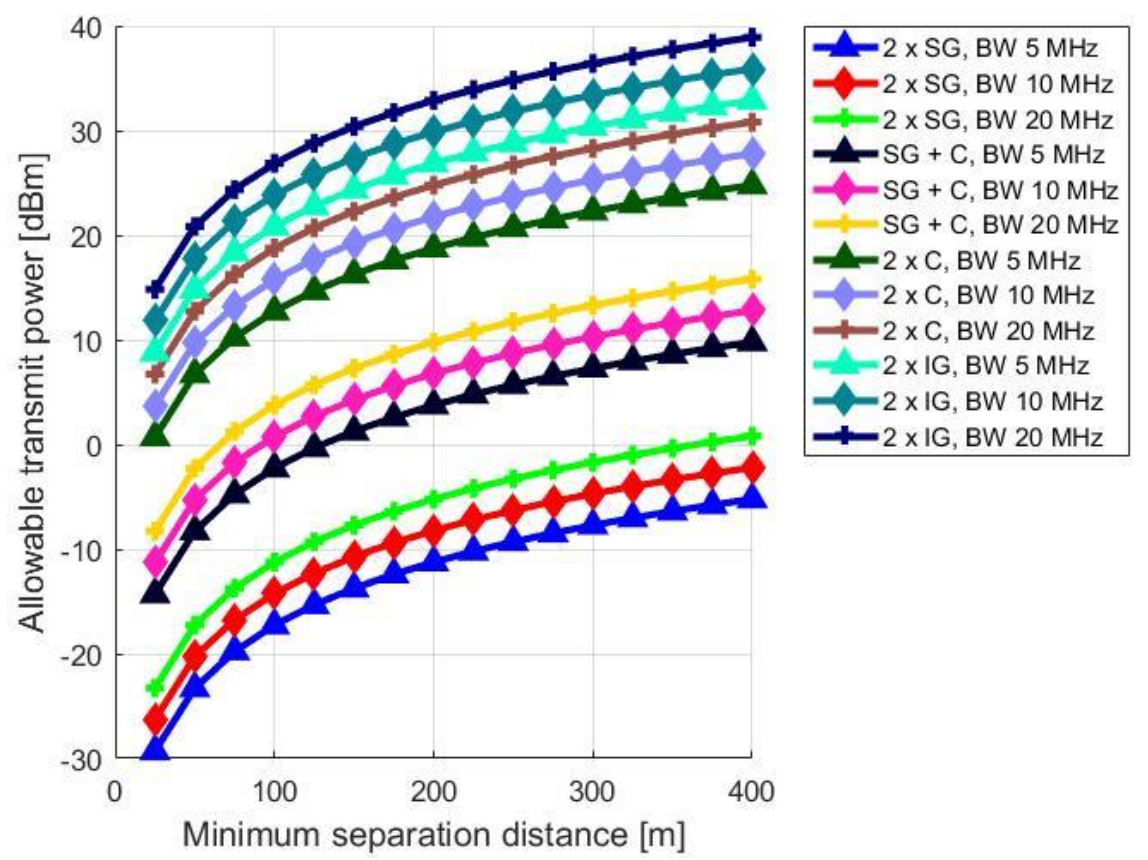

Fig. 2. Maximum allowable transmit power in co-channel case as a function of minimum separation distance with different types of building wall materials. 

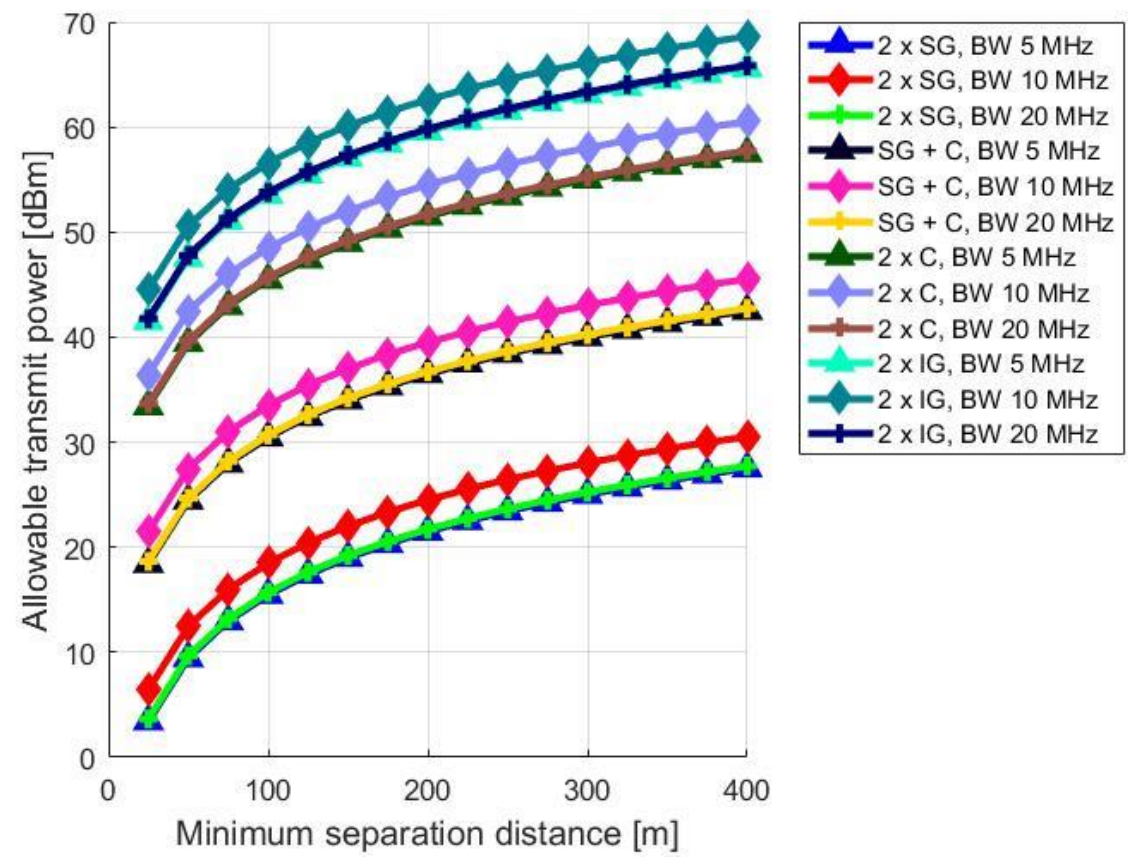

Fig. 3. Maximum allowable transmit power in adjacent channel case as a function of minimum separation distance with different types of building wall materials.

\section{$5 \quad$ Conclusions and future work}

Local small cell deployments will become an important operational mode in the future 5G networks especially when deployed in the higher carrier frequencies where coverages are inherently limited by the radio propagation characteristics. This calls for the development of new sharing-based spectrum authorization models for granting access right to deploy local $5 \mathrm{G}$ networks with a certain level of protection from harmful interference. Therefore, the characterization of the resulting interferences between local small cell deployments of different operators in co-channel and adjacent channel cases will be important to allow a wide variety of stakeholders become micro operators and to gain access to $5 \mathrm{G}$ spectrum to deploy and operate local small cell networks.

This paper has introduced a new deployment scenario for $5 \mathrm{G}$ small cells where two micro operators deploy local indoor small cell networks in separate buildings with locally issued spectrum micro licenses proposed in this paper. The presented study serves as the starting point for the more complicated interference characterizations in the $5 \mathrm{G}$ networks for spectrum sharing between small cell deployments with the new locally issued micro licenses. The example numerical results provided for the $3.5 \mathrm{GHz}$ band 
indicate that two networks of different micro operators cannot be deployed in the neighboring buildings if they share the same frequency band. If the micro operators' networks are deployed in the adjacent frequency bands, they could operate in close vicinity with respect to each other.

Future research is needed to introduce the micro operator concept with local spectrum micro licensing into the mainstream 5G development. In particular, the development of the entire micro licensing model including its rules and conditions as well as steps is currently an open topic. Future research is also needed in characterizing the interference from multiple base stations and mobile terminals between the different micro licensee deployments in co-channel and adjacent channel cases. As the carrier frequency significantly influences the propagation loss and resulting interference levels, it is important to properly characterize the frequency band and the systems in question to derive actual protection requirements. In determining the actual license conditions that guarantee the operators free from harmful interference, there is a need for accurate propagation loss modeling based on radio channel measurements that characterizes the different interference scenarios. Moreover, since the propagation loss is significantly larger at millimeter wave bands, there is a need to study the use of highly directive antennas at base station and model them properly in the interference studies at the higher frequency bands.

Acknowledgment. This work was supported by Tekes - the Finnish Funding Agency for Innovation in MOSSAF (Multi-Operator Spectrum Sharing for Future 5G Networks) and uO5G (Micro-Operator Concept for Boosting Local Service Delivery in $5 \mathrm{G})$ projects.

\section{References}

1. EC, "Communication from the Commission to the Parliament, the Council, the European Economic and Social Committee and the Committee of the Regions. Connectivity for a competitive digital single market - Towards a European gigabit society", European Commission, COM (2016) 587 final. 2016.

2. 5GPPP, "5G empowering vertical industries: Roadmap paper", The 5G Infrastructure Public Private Partnership (5GPPP). Brussels, 2016.

3. M. Matinmikko, M. Latva-aho, P. Ahokangas, S. Yrjölä, \& T. Koivumäki. Micro operators to boost local service delivery in 5G. Wireless Personal Communications, vol. 95, no. 1, pp. 69-82. 2017.

4. EC. "5G for Europe: An action plan", Communication from the Commission to the European Parliament, the Council, the European Economic and Social Committee and the Committee of the Regions. COM(2016) 588 Final, Brussels, 14th September 2016.

5. ECC, "Licensed shared access (LSA)", ECC Report 205. Electronic Communications Committee Committee (ECC) of European Conference of Postal and Telecommunications Administrations (CEPT). 2014. 
6. FCC, "Amendment of the Commission's Rules with regard to commercial operations in the $3550-3650 \mathrm{MHz}$ band, Order of reconsiderations and second report and order", Federal Communications Commission, FCC-16-55. 2016.

7. R. H. Tehrani, S. Vahid, D. Triantafyllopoulou, H. Lee \& K. Moessner, "Licensed spectrum sharing schemes for mobile operators: A survey and outlook," IEEE Communications Surveys \& Tutorials, vol. 18, no. 4, 2016, pp. 2591-2623.

8. A. K. Gupta, J. G. Andrews \& R. W. Heath, "On the feasibility of sharing spectrum licenses in mmWave cellular systems," IEEE Transactions on Communications, vol. 64, no. 9, 2016, pp. 3981-3995.

9. A. K. Gupta, A. Alkhateeb, J. G. Andrews, \& R. W. Heath. Restricted secondary licensing for mmWave cellular: How much gain can be obtained? IEEE Global Communications Conference (GLOBECOM), 4-8 December 2016, Washington, DC, USA, pp. 1-6.

10. J. Zander. Beyond the ultra-dense barrier: Paradigm shifts on the road beyond 1000x wireless capacity. IEEE Wireless Communications 2017 vol. 24, No. 3 pp. 96-102.

11. B. Singh, S. Hailu, K. Koufos, A. A. Dowhuszko, O. Tirkkonen, R. Jäntti \& R. Berry. Coordination protocol for inter-operator spectrum sharing in co-primary $5 \mathrm{G}$ small cell networks. IEEE Communications Magazine, 2015, Vol. 53, No. 7, pp. 34-40.

12. P. Ahokangas, S. Moqaddamerad, M. Matinmikko, A. Abouzeid, I. Atkova, J. Gomes, M. Iivari, "Future micro operators business models in 5G," In 6th International Conference on the Restructuring of the Global Economy (ROGE), University of Oxford, 20-21st June 2016, UK.

13. ITU-R M.2135, "Guidelines for evaluation of radio interface technologies for IMTAdvanced," Tech. Rep., 2009.

14. Aalto University, BUPT, CMCC, Nokia, NTT DOCOMO, New York University, Ericsson, Qualcomm, Huawei, Samsung, INTEL, University of Bristol, KT Corporation, and University of Southern California, " $5 \mathrm{G}$ channel model for bands up to $100 \mathrm{GHz}$, version 2.3," in 2nd Workshop on Mobile Communications in Higher Frequency Bands (MCHFB) in GLOBECOM, 6-10 Dec. 2015, San Diego, CA, USA, pp 1-102..

15. ECC Report 172, "Broadband wireless systems usage in 2300-2400 MHz", March 2012.

16. M. Jokinen, M. Mäkeläinen, T. Hänninen, M. Matinmikko, and M. Mustonen, "Minimum separation distance calculations for incumbent protection in LSA". In: Noguet D., Moessner K., Palicot J. (eds) Cognitive Radio Oriented Wireless Networks. CrownCom 2016. Lecture Notes of the Institute for Computer Sciences, Social Informatics and Telecommunications Engineering, vol 172. Springer, Cham.

17. ECC Report 203, "Least restrictive technical conditions suitable for mobile/fixed communication networks (MFCN), including IMT, in the frequency bands $3400-3600 \mathrm{MHz}$ and 3600$3800 \mathrm{MHz}$ ", March 2014.

18. 3GPP TR 38.901, "Study on channel model for frequencies from 0.5 to $100 \mathrm{GHz}$ " 3GPP, Tech. Rep., Mar. 2017.

19. 3GPP TS 36.104, "Base Station (BS) radio transmission and reception", 3GPP, Tech. Spec., Mar. 2017.

20. 3GPP TS 36.101, "User Equipment (UE) radio transmission and reception", 3GPP, Tech. Spec., Mar. 2017. 